\title{
The dependence of mass and environment on the secular processes of AGNs in terms of morphology, colour, and specific star-formation rate
}

\author{
M. Argudo-Fernández ${ }^{1,2}$, I. Lacerna ${ }^{3,4,5}$, and S. Duarte Puertas ${ }^{6}$ \\ 1 Centro de Astronomía (CITEVA), Universidad de Antofagasta, Avenida Angamos 601, Antofagasta, Chile \\ e-mail: maria.argudo@uantof.cl \\ ${ }^{2}$ Chinese Academy of Sciences South America Center for Astronomy, China-Chile Joint Center for Astronomy, \\ Camino El Observatorio 1515 Las Condes, Santiago, Chile \\ 3 Instituto de Astronomía, Universidad Católica del Norte, Av. Angamos 0610, Antofagasta, Chile \\ ${ }^{4}$ Instituto Milenio de Astrofísica, Av. Vicuna Mackenna 4860, Macul, Santiago, Chile \\ 5 Instituto de Astrofísica, Pontificia Universidad Católica de Chile, Av. V. Mackenna 4860, Santiago, Chile \\ ${ }^{6}$ Instituto de Astrofísica de Andalucía (CSIC) Apdo. 3004, 18080 Granada, Spain
}

Received 30 April 2018 / Accepted 2 October 2018

\begin{abstract}
Context. Galaxy mass and environment play a major role in the evolution of galaxies. In the transition from star-forming to quenched galaxies, active galactic nuclei (AGNs) also have a principal action therein. However, the connections between these three actors are still uncertain.

Aims. In this work we investigate the effects of stellar mass and the large-scale structure (LSS) environment on the fraction of optical nuclear activity in a population of isolated galaxies, where AGN would not be triggered by recent galaxy interactions or mergers. Methods. As a continuation of a previous work, we focus on isolated galaxies to study the effect of stellar mass and the LSS in terms of morphology (early- and late-type), colour (red and blue), and specific star-formation rate (quenched and star-forming). To explore where AGN activity is affected by the LSS, we separate galaxies into two groups, of low- and high mass, respectively, and use the tidal strength parameter to quantify the effects.

Results. We found that AGN is strongly affected by stellar mass in "active" galaxies (namely late-type, blue, and star-forming), but that mass has no influence on "quiescent" galaxies (namely early-type, red, and quenched), at least for masses down to $10^{10} M_{\odot}$. In relation to the LSS, we found an increase in the fraction of star-forming nuclei galaxies with denser LSS in low-mass star-forming and red isolated galaxies. Regarding AGN, we find a clear increase in the fraction of AGNs with denser environment in quenched and red isolated galaxies, independently of the stellar mass.

Conclusions. Active galactic nuclei activity appears to be "mass triggered" in active isolated galaxies. This means that AGN activity is independent of the intrinsic properties of the galaxies, but is dependent on their stellar mass. On the other hand, AGN activity appears to be "environment triggered" in quiescent isolated galaxies, where the fraction of AGNs as a function of specific star formation rate and colour increases from void regions to denser LSS, independently of stellar mass.
\end{abstract}

Key words. galaxies: active - galaxies: formation - galaxies: evolution - galaxies: star formation

\section{Introduction}

Galaxy mass and environment both play a major role in the evolution of galaxies from star-forming disk to passive spheroidal galaxies. This evolution can happen on a short timescale, where the star-formation activity is truncated by a discrete event, or could be due to a gradual increase of the average age of the stellar population (Casado et al. 2015). Two independent mechanisms, one related to stellar mass and another related to environment, have been proposed to explain this transition, generally referred to as "quenching" for local galaxies (Peng et al. 2010, 2012). In this regard, there would be an "environmental quenching" driven by galactic over-density (e.g. Hirschmann et al. 2014; Penny et al. 2016; Rodríguez del Pino et al. 2017), and "mass quenching", where the shut down of star formation occurs as part of a more dynamic process (e.g. Bluck et al. 2014; Fraser-McKelvie et al. 2016; Rowlands et al. 2018).

The quenching process has been broadly studied using diagnostic diagrams, such as the star formation rate (SFR) versus stellar-mass diagram, the specific star formation rate (sSFR) versus stellar-mass diagram, or the colour versus stellar-mass diagram (e.g., Brinchmann et al. 2004; Noeske et al. 2007; Rodighiero et al. 2011; Schawinski et al. 2014; Renzini \& Peng 2015; Duarte Puertas et al. 2017; Catalán-Torrecilla et al. 2017). In the SFR-stellar mass diagram, for example, active starforming galaxies define the main sequence at high values of the SFR, where starburst galaxies produced by merger processes appear as outlier galaxies to this main sequence (e.g. Rodighiero et al. 2011; Casado et al. 2015). Conversely, the quenched galaxies have higher stellar masses and lower SFR values. The use of these diagrams to study galaxies in different environments shows, for instance, that field galaxies are mainly located in starforming regions (the so-called blue cloud), whereas the group central galaxies are strongly biased to the passive regions (the so-called red sequence), which can be explained by the fact that the stellar-mass distributions of these two populations are different (Lacerna et al. 2014). The transition area between the two 
regions is commonly known as the green valley (e.g. Schawinski et al. 2014).

The principal mechanism responsible for quenching of star formation in galaxies is still unclear (e.g. Peng et al. 2015; Bait et al. 2017; Bremer et al. 2018; Coenda et al. 2018; NogueiraCavalcante et al. 2018; Smethurst et al. 2018). However, observations have led to the interpretation that active galactic nuclei (AGNs) form part of a plausible physical mechanism of the transformation of star-forming galaxies into passive galaxies (e.g. Bower et al. 2006; Heckman \& Best 2014; Cheung et al. 2016; Bongiorno et al. 2016; Smethurst et al. 2016; Penny et al. 2018). Accordingly, the green valley would be mainly populated by galaxies hosting an AGN (Terrazas et al. 2017). Nevertheless, the connections between AGNs, stellar mass, and environment are still not well understood (e.g. Harrison 2017; Cora et al. 2018).

Major mergers appear to be the main driver of high-luminosity AGN galaxies (Ellison et al. 2011; Ramos Almeida et al. 2012; Kaviraj et al. 2015; Manzer \& De Robertis 2014; Satyapal et al. 2014; Hong et al. 2015; Chiaberge et al. 2015). This result should be connected to galaxy mass since the AGN-driven feedback is usually proposed as a mechanism of quenching for massive galaxies after a major merger (e.g. Silk \& Rees 1998). Furthermore, AGN feedback is expected to play a major role in galaxies with massive halos (e.g. Gaspari et al. 2012), typically halo mass $M_{\mathrm{h}} \gtrsim 10^{12} M_{\odot}$ (Bower et al. 2006; Croton et al. 2006), which corresponds to approximately $M_{\star} \gtrsim 10^{10.5} M_{\odot}$. On the contrary, Sabater et al. $(2013,2015)$ suggest that the large-scale environment and galaxy interactions play a fundamental but indirect role in AGN activity, by influencing the gas supply, where the dependence on AGN luminosity is minimal.

The connection between environment and nuclear activity is not yet clear, however there is some consensus that secular processes may be much more important in driving black hole growth than previously assumed (McAlpine et al. 2015; Sabater et al. 2015), where an abundant supply of central cold gas, regardless of its origin, is the principal trigger of AGNs, which can be fed by secular processes. Black hole growth due to secular processes is dominant in isolated galaxies (Sabater et al. 2008; Hirschmann et al. 2013; Melnyk et al. 2015). The purpose of this study is therefore to identify the interplay of stellar mass and the large-scale structure environment (hereafter LSS) on driving AGN activity. We refer to "mass triggered" or "environment triggered" when the AGN is mostly triggered by stellar mass or environment, respectively, in a population of isolated galaxies where AGNs would not be triggered by recent galaxy interactions or mergers. With this work we are extending a previous study carried out by Argudo-Fernández et al. (2016). They observed different trends depending on galaxy mass. The fraction of low-mass isolated galaxies containing an AGN visible at optical wavelengths decreases from voids to denser regions, while the fraction of optical AGNs for high-mass isolated galaxies increases with denser large-scale environment. Since the prevalence of AGNs also depends on galaxy morphology (Moles et al. 1995; Schawinski et al. 2010; Hwang et al. 2012; Sabater et al. 2012), in this work we go one step further by exploring these trends as a function of galaxy morphology, colour, and sSFR. This work is complemented by a companion study (Lacerna et al. 2018) in which we focus on isolated elliptical galaxies to explore the connection of the large-scale environment in their integrated properties.

This study is organised as follows. In Sect. 2 we describe the sample of isolated galaxies used in this work as well as the selected AGN classification method, the morphology classifi- cation criteria to separate the sample into early- and late-type galaxies, the relations considered to separate galaxies between $\mathrm{red} / \mathrm{blue}$ and star-forming/quenched, and the parameters used to quantify the environment. We present our results in Sect. 3 and the associated discussion in Sect. 4. Finally, a summary and the main findings of the study are presented in Sect. 5. Throughout the study, a cosmology with $\Omega_{\Lambda 0}=0.7, \Omega_{\mathrm{m} 0}=0.3$, and $H_{0}=70 \mathrm{~km} \mathrm{~s}^{-1} \mathrm{Mpc}^{-1}$ is assumed.

\section{Data and methodology}

\subsection{Isolated galaxies}

Isolated galaxies represent a population of galaxies with minimized environmental evolutionary effects. We use galaxies of the SDSS-based catalogue of isolated galaxies (hereafter SIG; Argudo-Fernández et al. 2015b). These SIG galaxies are selected from the tenth data release of the Sloan Digital Sky Survey (SDSS-DR10; Ahn et al. 2014) in a volume-limited sample redshift range $0.005 \leq z \leq 0.080$, with $11 \leq m_{r} \leq 15.7$, where $m_{r}$ is the SDSS model magnitude in the $r$-band. The SIG is composed of 3702 isolated galaxies, which represents about $11 \%$ of the galaxies in the local universe $(z \leq 0.080$; Argudo-Fernández et al. 2015b).

Under a three-dimensional (3D) isolation criterion, SIG galaxies are identified as those with no neighbours in a volume of $1 \mathrm{Mpc}$ projected distance within a line-of-sight velocity difference of $\Delta v \leq 500 \mathrm{~km} \mathrm{~s}^{-1}$, and that are at least two orders of magnitude fainter within the range of spectroscopic completeness of the SDSS main galaxy sample at $m_{r \text {,Petrosian }}<17.77$ mag (Strauss et al. 2002). See Argudo-Fernández et al. (2015b) for further details.

\subsection{Stellar masses and AGN classification}

We used published stellar masses and AGN classifications for galaxies in the SDSS-DR7 from Sabater et al. (2013); hereafter SBA13 classification. In particular, we focus on optically selected nuclear activity.

Total stellar masses and AGN classification in SBA13 were drawn from Kauffmann et al. (2003b) and from Baldwin, Phillips, and Terlevich (BPT) diagnostic (Baldwin et al. 1981; Kauffmann et al. 2003a), respectively. The information about optical spectra, such as the corrected emission-line fluxes necessary to build BPT diagrams, is drawn from the Max Plank Institute for Astrophysics and John Hopkins University (MPAJHU ${ }^{1}$; Kauffmann et al. 2003b; Tremonti et al. 2004; Salim et al. 2007) added value catalogue (Brinchmann et al. 2004).

The fraction of AGNs depends strongly on stellar mass (Kauffmann et al. 2003a; Peng et al. 2010, 2012). Therefore, to have enough galaxies in each mass bin, we considered galaxies with a stellar mass within the range $10.0 \leq \log \left(M_{\star}\right) \leq 11.4 M_{\odot}$ (see the three panels in Fig. 1). There are 2299 SIG galaxies in this stellar mass range that have an available SBA13 classification. The total number of these galaxies classified by morphology, colour, and sSFR (when classification is possible; see following sub-sections) is shown in the first row of Table 1 (see Sects. 2.3 and 2.4). We note that there may be repeated galaxies in each classification column; for instance some SIG late-type galaxies can also be classified as blue galaxies.

Following SBA13, AGN classification includes transition objects (TO), Seyfert (Seyfert 1 not included), and low-

Available at http: //www .mpa-garching.mpg.de/SDSS/DR7/ 

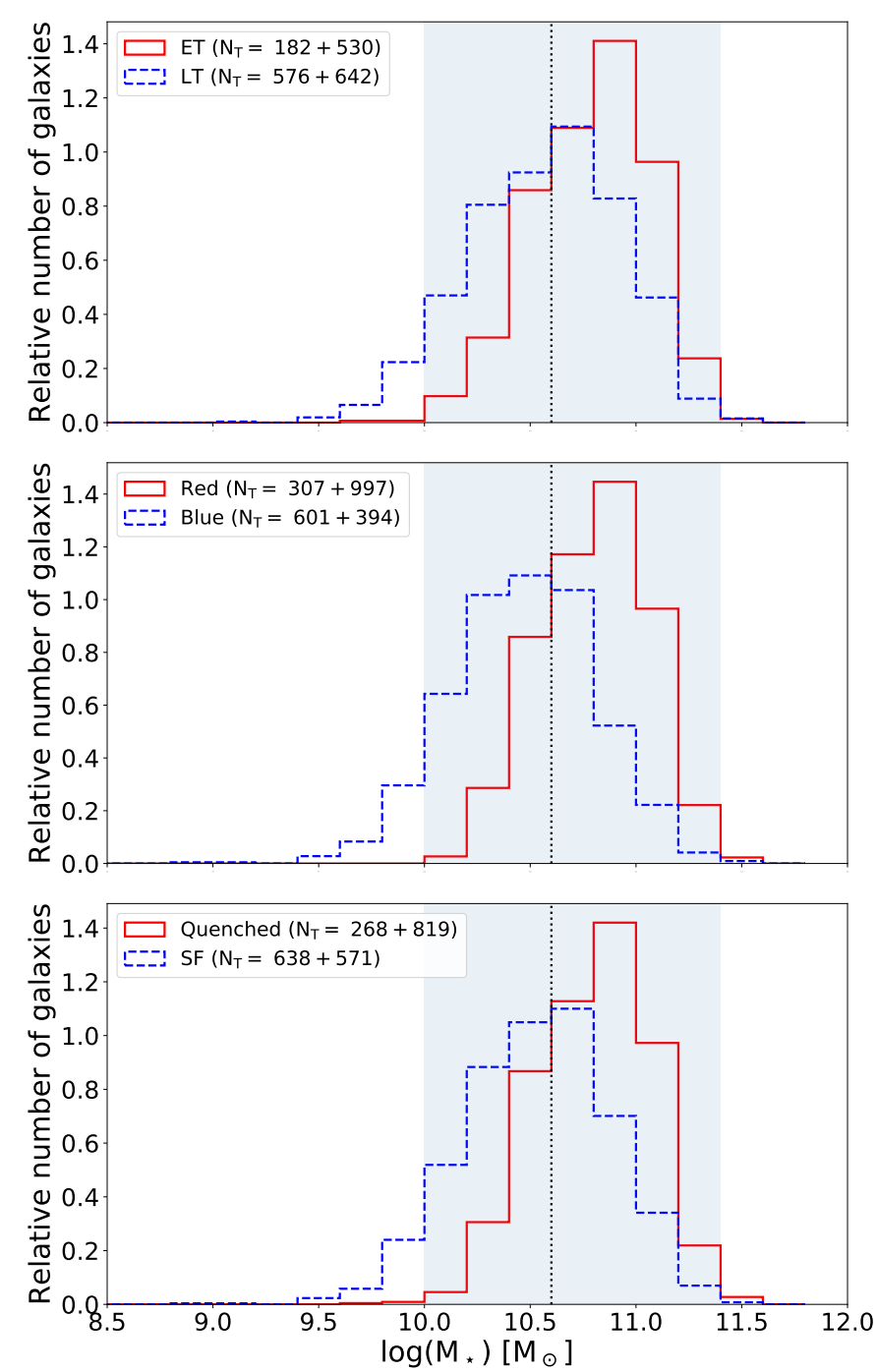

Fig. 1. Distribution of the stellar masses for galaxies in the SIG sample according to their morphology (red solid line for early-type galaxies and blue dashed line for late-type galaxies in the top panel), colour (red solid line for red galaxies and blue dashed line for blue galaxies in the middle panel), and sSFR (red solid line for quenched galaxies and blue dashed line for star-forming galaxies in the lower panel). The shaded area in the figures corresponds to the selected stellar mass range for this study at $10.0 \leq \log \left(M_{\star}\right) \leq 11.4 M_{\odot}$. The vertical black dotted line corresponds to the selected mass cut to distinguish between low-mass and high-mass galaxies at $\log \left(M_{\star}\right)=10.6 M_{\odot}$. The total number of galaxies at each side of the mass cut is indicated in the corresponding legend.

ionization nuclear emission-line region (LINER; Heckman 1980) galaxies. To have a statistically significant number of galaxies when dividing our sample by morphology, colour, and sSFR, we only separate nuclear activity into AGN, star-forming nuclei (SFN), and passive galaxies (in cases where no nuclear activity is detected). To check that our results are not biased by this separation, we also explore the observed trends considering the different AGN subtypes in Appendix A. The number of galaxies classified as having each type of nuclear activity is also shown in Table 1.

We also note that, for the reason above, we do not impose any limit on the $\left[\mathrm{O}_{\mathrm{III}}\right]_{5007}$ emission line luminosity. We therefore need to take into account that some low-luminosity AGNs (usually LINERs) at higher redshifts could be classified as passive if their emission is not strong enough to be detected. Addi-
Table 1. Number of galaxies in each subsample.

\begin{tabular}{lcccccc}
\hline \hline Type & ET & LT & red & blue & quenched & SF \\
\hline Total & 712 & 1218 & 1304 & 995 & 1087 & 1209 \\
& & & & & & \\
AGN & 362 & 586 & 768 & 385 & 611 & 541 \\
TO & 121 & 332 & 299 & 249 & 138 & 409 \\
Seyfert & 37 & 65 & 77 & 46 & 62 & 60 \\
LINER & 204 & 189 & 392 & 90 & 410 & 72 \\
SFN & 44 & 462 & 101 & 486 & 8 & 577 \\
Passive & 306 & 170 & 435 & 124 & 468 & 91 \\
\hline
\end{tabular}

Notes. Meaning of the different types. ET: early-type galaxies as defined in Sect. 2.3; LT: late-type galaxies as defined in Sect. 2.3; red: red galaxies as defined in Sect. 2.4; blue: blue galaxies as defined in Sect. 2.4; quenched: centrally quenched galaxies as defined in Sect. 2.4; SF: centrally star-forming galaxies as defined in Sect. 2.4. Total: total number of galaxies in each subsample; AGN: galaxies classified as LINER, Seyfert, or transition objects (TO); SFN: star-forming nuclei galaxies; passive: galaxies with no optical nuclear activity.

tionally, we discuss our results when considering a different AGN classification, composed of TO and Seyfert galaxies in Sect. 4.1.

\subsection{Morphology}

Galaxy morphology is another fundamental property used to understand galaxy evolution. In the hierarchical galaxy formation scenario, major mergers is the prevailing mechanism of formation of early-type galaxies, which commonly happen in dense regions (e.g. Hernquist 1993; Kauffmann 1996; Tutukov et al. 2007; Schawinski et al. 2014). Therefore, it is expected that a high fraction of the population of isolated galaxies is composed of late-type galaxies (Dressler 1980; Hernández-Toledo et al. 2010; Lacerna et al. 2014; Vulcani et al. 2015). A previous analysis of AGN activity in isolated elliptical galaxies in the local universe was performed in Lacerna et al. (2016). In this paper, we aim to investigate the AGN activity for both isolated earlytype galaxies and isolated late-type galaxies.

We used the automated morphology classification for a representative sample of galaxies developed by Huertas-Company et al. (2011). They provide a catalogue with a detailed, automated computational morphological classification for 698420 galaxies in the SDSS-DR7 (Abazajian et al. 2009) spectroscopic catalogue with redshift less than 0.25 and $m_{r} \leq 16$ mag. Huertas-Company et al. (2011) divided the morphologies into four classes (E, S0, Sab, and Scd) and a broader early-type class (E or S0). Their automated method associates each galaxy with a probability of belonging to each one of those categories. We considered the probability of being an early-type galaxy $\left(p_{\text {Early }}\right)$ to separate between early- and late-type galaxies.

There are 2293 SIG galaxies with morphological classification, in the stellar-mass range considered in this study and with available SBA13 classification. Of them there are 712 early-type galaxies (when $p_{\text {Early }}>0.7$ ) and 1218 late-type galaxies (when $\left.p_{\text {Early }}<0.3\right)$.

\subsection{Colour and specific star formation rate}

We also aim to study the fraction of AGN, SFN, and passive galaxies as a function of environment in terms of galaxy properties such as colour and sSFR. We use the relation found by Lacerna et al. (2014) to separate red and blue galaxies, specifically 
$(g-i)=0.16\left[\log \left(M_{\star}\right)-10.31\right]+1.05$,

where the magnitudes $g$ and $i$ were taken from the SDSS database with extinction corrected modelMag magnitudes (dered parameter), and the stellar mass is in units of $M_{\odot}$. We appended/added to our catalogues the photometric information/data from SDSS-DR12 (Alam et al. 2015). With this method we were able to classify all the SIG galaxies in our study in terms of colour. The SIG galaxies lying below the relation in the $(g-i)-\log \left(M_{\star}\right)$ diagram are classified as blue (995 SIG galaxies), meanwhile SIG galaxies above the line are classified as red (1304 SIG galaxies).

Likewise, we use the relation provided by Lacerna et al. (2014) to separate quenched and star-forming galaxies in terms of their sSFR, that is,

$\log (\mathrm{sSFR})=-0.65\left[\log \left(M_{\star}\right)-10.31\right]-10.87$,

where sSFR is in units of $\mathrm{yr}^{-1}$ and the stellar mass is in units of $M_{\odot}$. The sSFR has been obtained from the MPA-JHU catalogue using a spectrophotometric synthesis fitting model. See Lacerna et al. (2014) for further details of these two relations. Following a similar criteria, galaxies above the relation are classified as star-forming (SF, 1209 SIG galaxies) and galaxies below as quenched (1087 SIG galaxies). There are three SIG galaxies with undefined SSFR, so we were not able to classify them.

We note that AGN classification based purely on emissionline BPT diagrams (as used in SBA13) may be affected by uncertainties (Sabater et al. 2012), where misclassified galaxies could be classified as LINERs or Seyferts. These uncertainties are specially important for massive main-sequence local galaxies that might be misclassified as passive (Rosario et al. 2016). ArgudoFernández et al. (2016) checked that less than $4 \%$ of the total number of galaxies in the SIG would be affected.

At the same time, the selection of SFN galaxies is based on spectra obtained in the central fibre of the SDSS-DR7, which can be affected by AGNs. We can obtain a more reliable selection of AGN and SFN galaxies combining the two methods (BPT diagrams and synthesis fitting model to the spectra). Therefore, we study the fraction of AGN/SFN/passive galaxies as a function of the population of SF/quenched galaxies. We also note that a size-limited fibre leads to a different galaxy area coverage, and therefore fibre may cover some part of the disk for more distant galaxies. According to Kauffmann et al. (2003b), galaxies classified as SFN do not have more than a $1 \%$ contribution of AGNs; we are therefore confident that we are not affected by contamination of AGNs in galaxies classified as SFN. Nevertheless, we may misclassify galaxies with a star-forming disk as AGNs. Given that galaxies with SBA13 classification are restricted to the local and narrow redshift range $0.03 \leq z \leq 0.08$, we do not expect a bias in our results caused by AGN misclassification or even possible redshift evolution.

In summary, the number of AGN, SFN, and passive galaxies in terms of morphology, colour, and SSFR are collected in Table 1. As expected, there are small numbers of early-type, red, and quenched SFN SIG galaxies, respectively. Similarly, the numbers of late-type, blue, and star-forming isolated passive galaxies are also small.

\subsection{Quantification of the environment}

The public SIG catalogue also provides the isolation degree for SIG galaxies in terms of their large-scale environment (ArgudoFernández et al. 2015b). They use the tidal strength parameter
(Verley et al. 2007; Sabater et al. 2013; Argudo-Fernández et al. $2013,2014)$ to quantify the influence of the neighbour galaxies in their LSS, which are the galaxies lying within a volume of $5 \mathrm{Mpc}$ projected distance and with line-of-sight velocity difference of $\Delta v \leq 500 \mathrm{~km} \mathrm{~s}^{-1}$.

To study the influence of the LSS on the AGN fraction for SIG galaxies, we therefore selected the $Q_{\text {LSs }}$ parameter (Eq. (3)), that is, the tidal strength exerted by all the galaxies in the LSS. Then, for each galaxy, $i$, in the LSS at a projected distance $d_{\mathrm{LSS}_{i}}$ and stellar mass $M_{\mathrm{LSS}}{ }^{2}$, the total tidal strength on the SIG galaxy is:

$Q_{\mathrm{LSS}} \equiv \log \left(\sum_{i} \frac{M_{\mathrm{LSS}_{i}}}{M}\left(\frac{D}{d_{\mathrm{LSS}_{i}}}\right)^{3}\right)$

where $M$ and $D^{3}$ are the stellar mass and the estimated diameter of the SIG galaxy, respectively.

The greater the value of $Q_{\mathrm{LSs}}$, the less isolated the galaxy is from external influence. In fact, using the visualisation tool LSSGalPY (Argudo-Fernández et al. 2015a, 2017), ArgudoFernández et al. (2016) checked that SIG galaxies with low values of $Q_{\mathrm{LSS}}$ are generally located in voids $\left(Q_{\mathrm{LSS}}<-5.5\right)$, and those with higher values are more closely linked to denser structures, for example the outskirts of clusters $\left(Q_{\mathrm{LSS}}>-4.5\right)$. In this regard, it is appropriate to note SIG galaxies mainly belong to the outer parts of filaments, walls, and clusters, and generally differ from the void population of galaxies, where only one-third of SIG galaxies are located in voids (Argudo-Fernández et al. 2015b).

\section{Results}

\subsection{AGN prevalence in isolated galaxies}

We compare the relative fraction of SFN, AGN, and passive galaxies with respect to the stellar mass in the SIG, as a function of morphology, colour, and sSFR. Since there is a strong dependence of AGN fraction with stellar mass, it is recommended to carry out a separate study using different stellar mass bins SBA13. According to the distributions shown in Fig. 1, we separate into seven stellar mass bins from $\log \left(M_{\star}\right)=10.0 M_{\odot}$ to $\log \left(M_{\star}\right)=11.4 M_{\odot}$.

The left panel in Fig. 2 shows that the fractions of late-type, blue, and star-forming SIG galaxies containing an SFN decrease with stellar mass, where the fraction for late-type galaxies is slightly steeper. On the contrary, the fractions of the same galaxies that contain an AGN increase with stellar mass (middle panel in Fig. 2). The fraction of passive galaxies also increases but the tendency is less steep, where the fraction for star-forming isolated galaxies is the smoothest (right panel in Fig. 2). The same trend of AGN galaxies increasing with stellar mass is observed if we separate late-type, blue, and star-forming SIG galaxies into TOs, Seyferts, and LINERs (see Fig. A.1).

The fractions of SFN, AGN, and passive galaxies in earlytype, red, and quenched SIG galaxies are roughly constant with stellar mass, except for the first bin, where the number of these galaxies at low stellar mass is small.

2 Stellar masses for galaxies in the LSS were calculated by fitting the SED on the five SDSS bands using the routine kcorrect (Blanton \& Roweis 2007).

${ }^{3} D=2 \alpha r_{90}$ (Argudo-Fernández et al. 2013), where $r_{90}$, the Petrosian radius containing $90 \%$ of the total flux of the galaxy in the $r$-band, is scaled by a factor $\alpha=1.43$ to recover the $D_{25}$, defined by the $\mu_{B}(B)=25.0 \mathrm{mag} / \operatorname{arcsec}^{2}$ isophote. 
M. Argudo-Fernández et al.: The dependence of mass and environment on the secular processes of AGNs

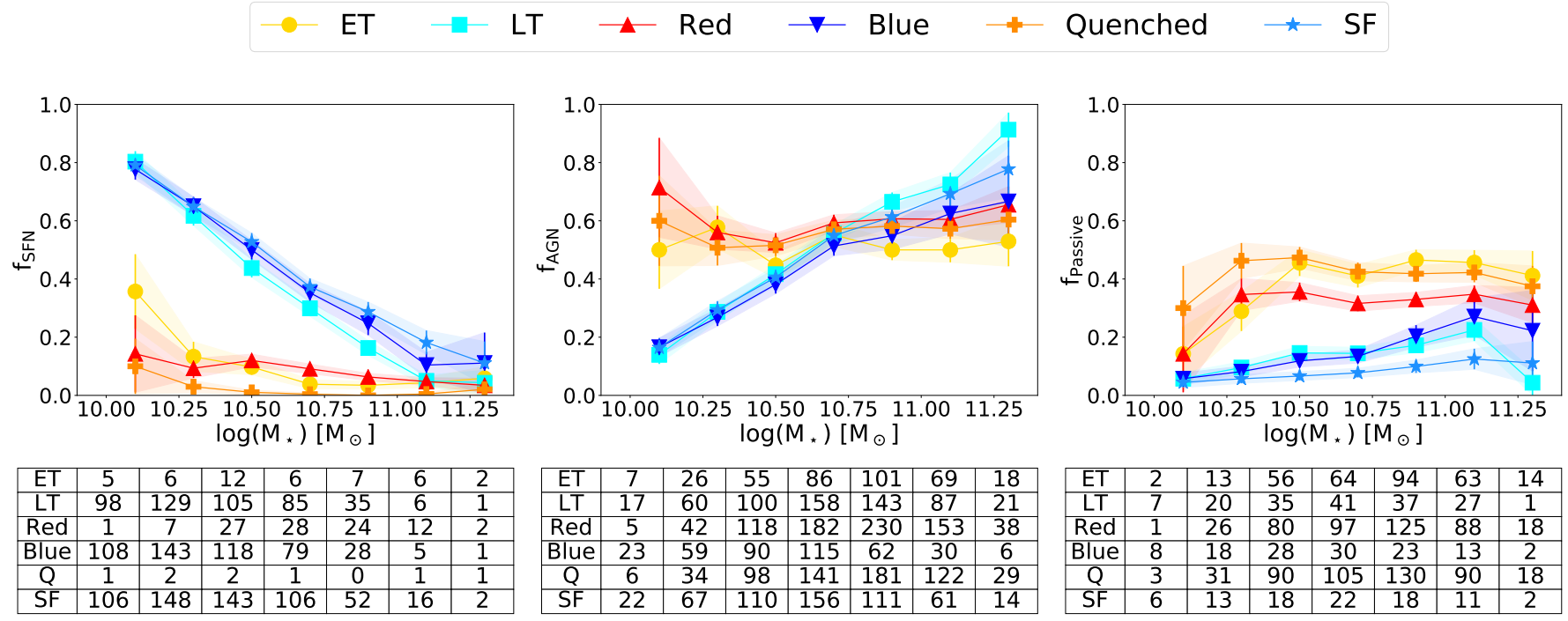

Fig. 2. Fraction of SFN (left panel), optical AGN (middle panel), and passive galaxies (right panel) with respect to stellar mass. The fraction in early-type $\left(N_{T}=712\right)$, red $\left(N_{T}=1304\right)$, and quenched $\left(N_{T}=1087\right)$ SIG galaxies is depicted by yellow circles, red triangles, and orange plus symbols, respectively. Cyan squares correspond to the fraction in late-type SIG galaxies $\left(N_{T}=1218\right)$, blue inverted triangles for blue SIG galaxies $\left(N_{T}=995\right)$, and light blue stars for star-forming SIG galaxies $\left(N_{T}=1209\right)$. The number of galaxies in each stellar mass bin is shown in tables for each sample at the bottom of each panel. Error bars are given by considering binomial distribution.

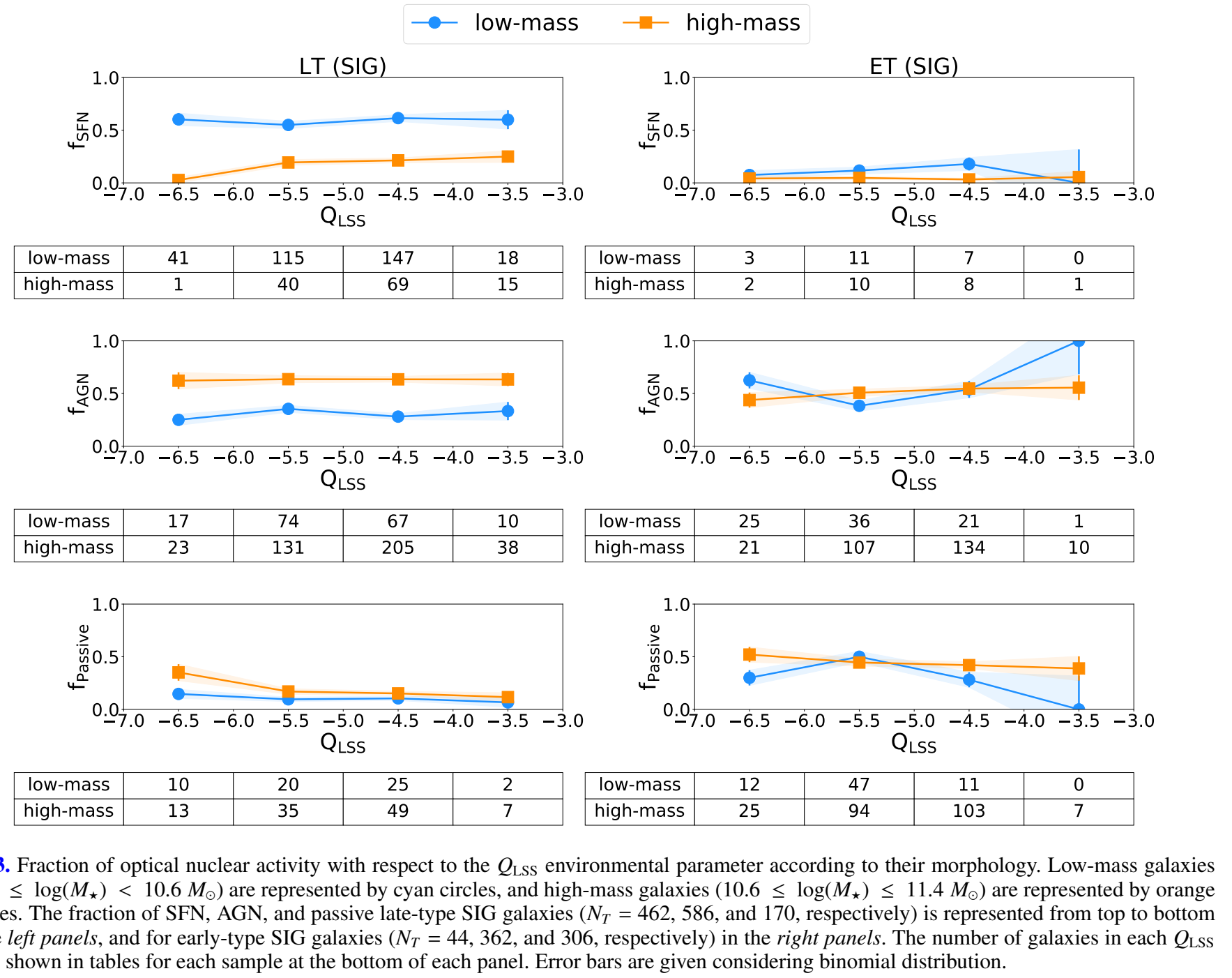



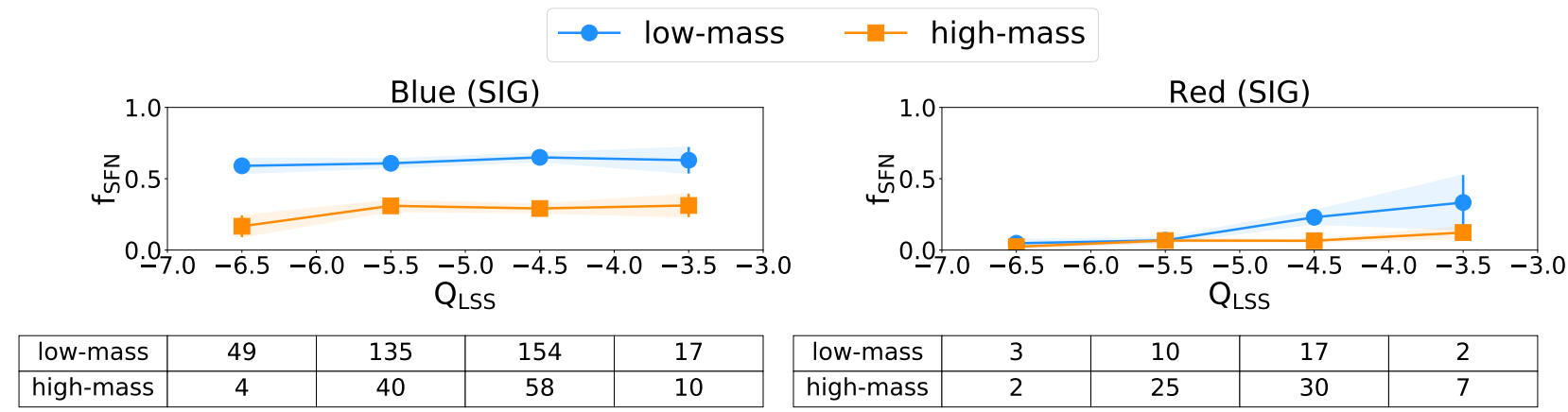

\begin{tabular}{|c|c|c|c|c|}
\hline low-mass & 49 & 135 & 154 & 17 \\
\hline high-mass & 4 & 40 & 58 & 10 \\
\hline
\end{tabular}

\begin{tabular}{|c|c|c|c|c|}
\hline low-mass & 3 & 10 & 17 & 2 \\
\hline high-mass & 2 & 25 & 30 & 7 \\
\hline
\end{tabular}
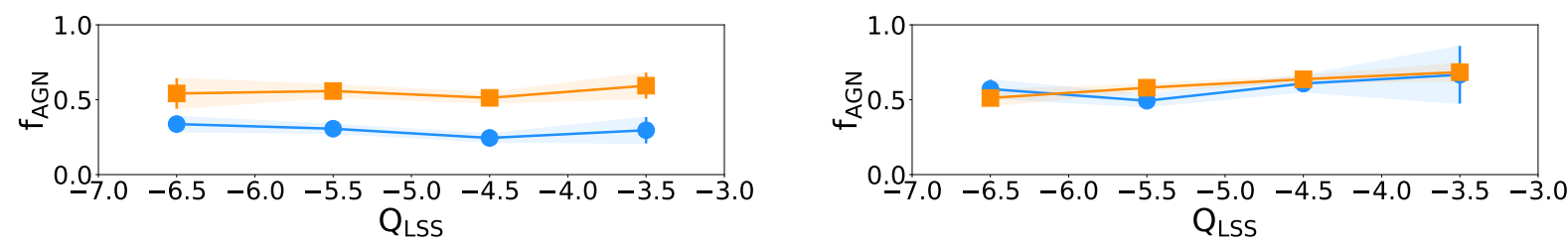

\begin{tabular}{|c|c|c|c|c|}
\hline low-mass & 28 & 68 & 58 & 8 \\
\hline high-mass & 13 & 72 & 102 & 19 \\
\hline
\end{tabular}

\begin{tabular}{|c|c|c|c|c|}
\hline low-mass & 36 & 72 & 45 & 4 \\
\hline high-mass & 44 & 217 & 293 & 39 \\
\hline
\end{tabular}
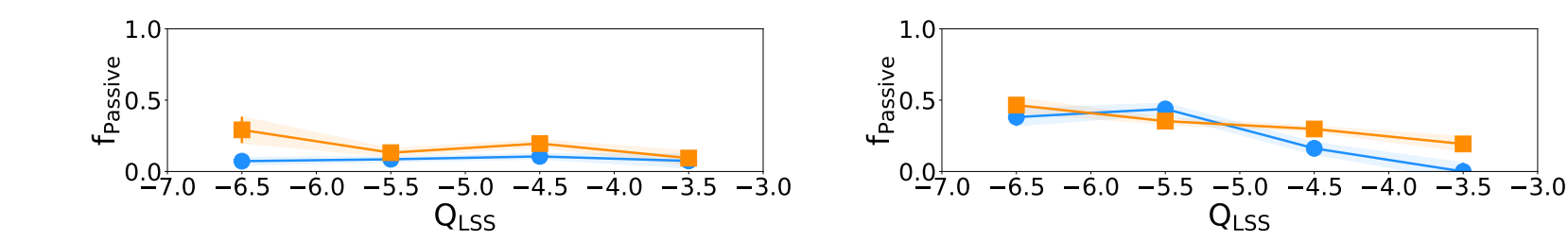

\begin{tabular}{|c|c|c|c|c|}
\hline low-mass & 6 & 19 & 25 & 2 \\
\hline high-mass & 7 & 17 & 39 & 3 \\
\hline
\end{tabular}

\begin{tabular}{|c|c|c|c|c|}
\hline low-mass & 24 & 64 & 12 & 0 \\
\hline high-mass & 40 & 132 & 137 & 11 \\
\hline
\end{tabular}

Fig. 4. Fraction of optical nuclear activity with respect to the $Q_{\text {LSs }}$ environmental parameter according to their colour. Low-mass galaxies $\left(10.0 \leq \log \left(M_{\star}\right)<10.6 M_{\odot}\right)$ are represented by cyan circles, and high-mass galaxies $\left(10.6 \leq \log \left(M_{\star}\right) \leq 11.4 M_{\odot}\right)$ are represented by orange squares. The fraction of SFN, optical AGN, and passive blue SIG galaxies ( $N_{T}=486,385$, and 124, respectively) is represented from top to bottom in the left panels, and for red SIG galaxies $\left(N_{T}=101,768\right.$, and 435 , respectively) in the right panels. The number of galaxies in each $Q_{\mathrm{LSS}}$ bin is shown in tables for each sample at the bottom of each panel. Error bars are given considering binomial distribution.

As shown in the left panel in Fig. 2, in general the fractions of late-type, blue, and star-forming SIG galaxies classified as SFNs in each stellar mass bin are larger than the fractions of earlytype, red, quenched SIG galaxies, but are smaller for galaxies classified as passive, as shown in the right panel. In the case of galaxies classified as AGNs, the fractions of late-type, blue, and star-forming galaxies are in general higher than or equal to those of early-type, red, and quenched SIG galaxies at stellar masses of $\log \left(M_{\star}\right)>10.6 M_{\odot}$.

We note that the observed trends may be affected by the low number of early-type, red, and quenched galaxies at low stellar mass, as well as low numbers of late-type, blue, and star-forming galaxies at high stellar mass (see also the stellar mass distributions in Fig. 1). For that reason we focus only on general trends observed in bins with larger statistics.

\subsection{Dependence on the LSS environment}

As introduced in Sect. 2.5, we use the $Q_{\text {LSS }}$ to quantify the effect of the LSS on the fractions of SFN, AGN, and passive galaxies in the SIG. To take into account the effect of the mass and to obtain a sufficient sample size for robust statistical analyses, we divide the samples into two stellar mass bins: low-mass galaxies $\left(10.0 \leq \log \left(M_{\star}\right)<10.6 M_{\odot}\right)$ and high-mass galaxies $\left(10.6 \leq \log \left(M_{\star}\right) \leq 11.4 M_{\odot}\right)$. We selected this mass cut value of $\log \left(M_{\star}\right)=10.6 M_{\odot}$ to have a similar number of galaxies at both sides of the distributions (see Fig. 1) and also it is approximately the mass value where the fraction of AGNs for early-type, red, and quenched SIG galaxies matches the fraction of AGNs for late-type, blue, star-forming SIG galaxies.

Figure 3 shows the fraction of optical nuclear activity, segregated into low- and high-mass bins, with respect to $Q_{\mathrm{LSS}}$, for SIG galaxies as a function of their morphology. Higher values of the $Q_{\text {LSS }}$ correspond to a stronger interaction with the LSS. Similarly, Figs. 4 and 5 show the fractions of SFN, AGN, and passive SIG galaxies in terms of colour and sSFR, respectively.

In general, there is no clear dependence on the LSS environment in the nuclear activity for SIG galaxies in terms of morphology and colour, although there is a trend for high-mass SIG galaxies to increase with denser LSS environment. There are some trends in terms of sSFR classification, which are discussed in Sect. 4, as well as other general trends.

\section{Discussion}

\subsection{AGN prevalence in isolated galaxies}

The prevalence of AGNs is known to depend strongly on the mass of the host galaxy (Kauffmann et al. 2003a, 2004; Best et al. 2005). Following on from the previous work in Argudo-Fernández et al. (2016), we control stellar mass restricting the galaxies to the stellar mass range 

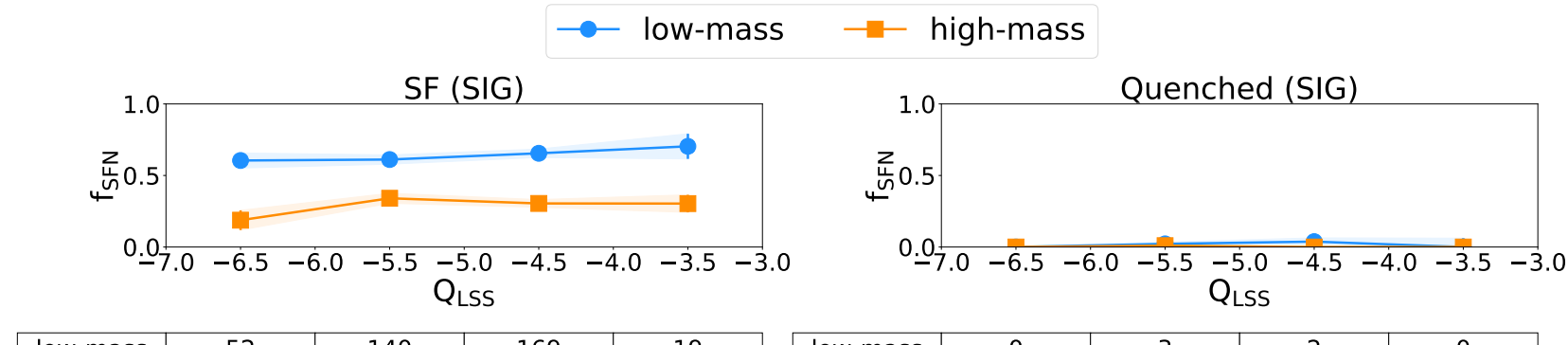

\begin{tabular}{|c|c|c|c|c|}
\hline low-mass & 52 & 140 & 169 & 19 \\
\hline high-mass & 6 & 62 & 88 & 17 \\
\hline
\end{tabular}

\begin{tabular}{|c|l|l|l|l|}
\hline low-mass & 0 & 3 & 2 & 0 \\
\hline high-mass & 0 & 3 & 0 & 0 \\
\hline
\end{tabular}
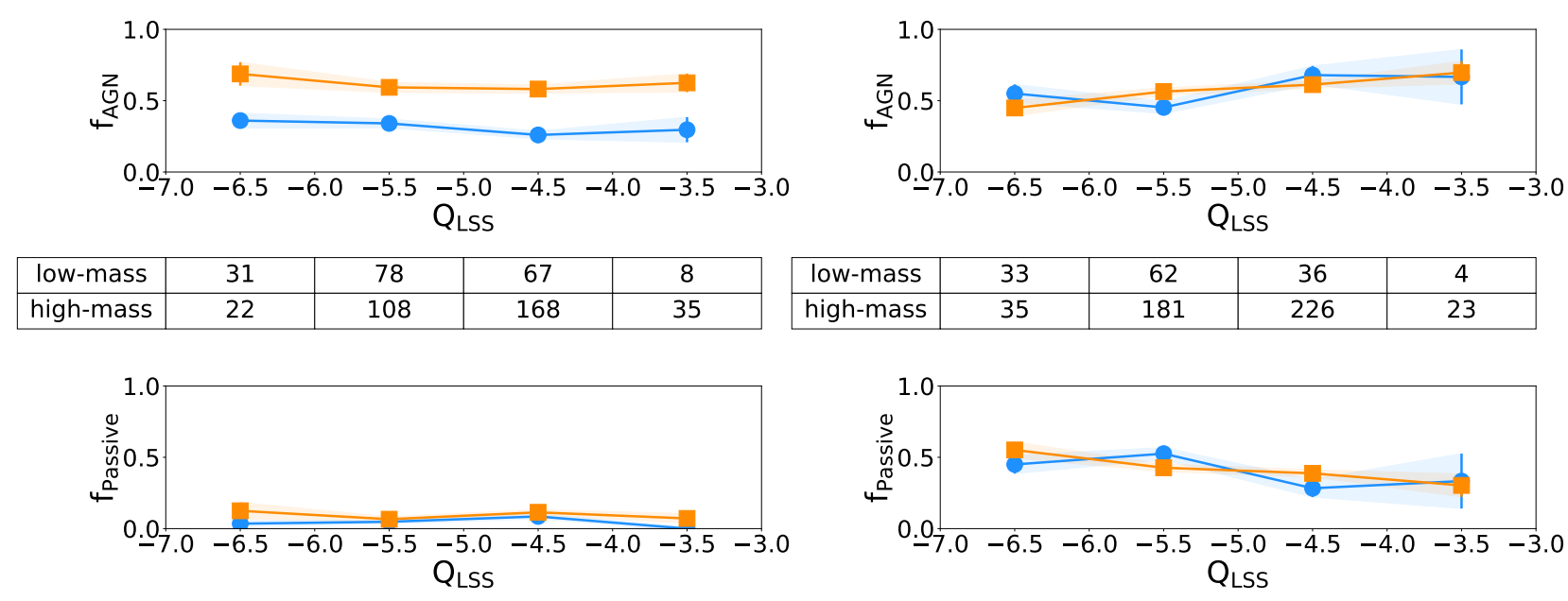

\begin{tabular}{|c|c|c|c|c|}
\hline low-mass & 33 & 62 & 36 & 4 \\
\hline high-mass & 35 & 181 & 226 & 23 \\
\hline
\end{tabular}

\begin{tabular}{|c|l|l|l|l|}
\hline low-mass & 3 & 11 & 22 & 0 \\
\hline high-mass & 4 & 12 & 33 & 4 \\
\hline
\end{tabular}

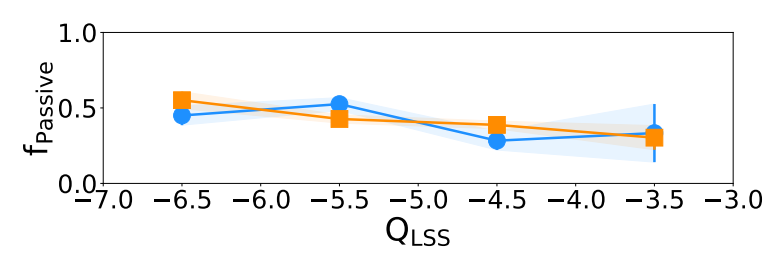

\begin{tabular}{|c|c|c|c|c|}
\hline low-mass & 27 & 72 & 15 & 2 \\
\hline high-mass & 43 & 137 & 143 & 10 \\
\hline
\end{tabular}

Fig. 5. Fraction of optical nuclear activity with respect to the $Q_{\text {LSS }}$ environmental parameter according to their sSFR status. Low-mass galaxies $\left(10.0 \leq \log \left(M_{\star}\right)<10.6 M_{\odot}\right)$ are represented by cyan circles, and high-mass galaxies $\left(10.6 \leq \log \left(M_{\star}\right) \leq 11.4 M_{\odot}\right)$ are represented by orange squares. The fraction of SFN, optical AGN, and passive star forming SIG galaxies $\left(N_{T}=577,541\right.$, and 91 , respectively) is represented from top to bottom in the left panels, and for quenched SIG galaxies $\left(N_{T}=8,611\right.$, and 468 , respectively) in the right panels. The number of galaxies in each $Q_{\mathrm{LSS}}$ bin is shown in tables for each sample at the bottom of each panel. Error bars are given considering binomial distribution.

$10.0 \leq \log \left(M_{\star}\right) \leq 11.4 M_{\odot}$. They found that, for any of the galaxy samples considered in their study, the fraction of SFNs decreases with stellar mass, and the fractions of AGN and passive galaxies increase with higher stellar mass (where the trend is steeper for AGNs). In the present work we explore these trends in isolated galaxies as a function of their morphology, colour, and sSFR.

As is shown in Fig. 2, the previous trends are only observed in late-type, blue, and star-forming isolated galaxies. However, the fractions for early-type, red, and quenched isolated galaxies are roughly constant and independent of the stellar mass. This means that once an isolated galaxy is in a quiescent state (evolved, without forming new stars), there is an approximately 50 to $60 \%$ probability that the galaxy hosts an AGN and about $40 \%$ probability that is a passive galaxy, independently of its stellar mass. Argudo-Fernández et al. (2016) did not observe this bimodality since most of the SIG (about 75\%) is composed of late-type galaxies, in agreement with the morphology-density relation.

According to the central panel in Fig. 2, there is a higher fraction of AGNs in low-mass early-type, red, and quenched isolated galaxies than there is in low-mass late-type, blue, and star-forming isolated galaxies, in agreement with Sabater et al. (2012) and Hernández-Ibarra et al. (2016), which suggests that a bulge and a large gas reservoir are both essential to trigger optical nuclear activity. Once an isolated galaxy reaches a stellar mass of about $\log \left(M_{\star}\right) \sim 10.6 M_{\odot}$, the probability that it hosts an AGN is independent of its morphology, colour, or sSFR. At higher stellar mass, the fraction of AGNs in late-type, blue, and star-forming isolated galaxies is larger. This may be affected by the fact that galaxies with stellar masses $\log \left(M_{\star}\right)>10.5 M_{\odot}$ may present large bulges, and therefore they show lower star formation and favour AGN classification in a BPT diagram (Brinchmann et al. 2004; Richards et al. 2016). In fact Duarte Puertas et al. (2017) observe a decrease of the SFR in galaxies with $\log \left(M_{\star}\right)>10.6 M_{\odot}$.

For consistency with Argudo-Fernández et al. (2016), and to have a statistically meaningful number of galaxies, we consider TO, Seyfert, and LINER as AGN galaxies following also the work of Sabater et al. (2013). However, recent studies of the spatially resolved nebular emission in nearby galaxies using Integral Field Unit (IFU) data suggest that LINER-like emission is more closely related to evolved stars and not directly related to nuclear ionization (e.g. Singh et al. 2013; Belfiore et al. 2016). In this regard, we explore the effect of considering LINER galaxies in the AGN classification.

Similar to the central panel in Fig. 2, Fig. 6 shows the fraction of AGN when considering only Seyfert and TO galaxies. We observe similar trends under this consideration, that is, the fraction of AGNs increases with stellar mass in late-type, blue, 

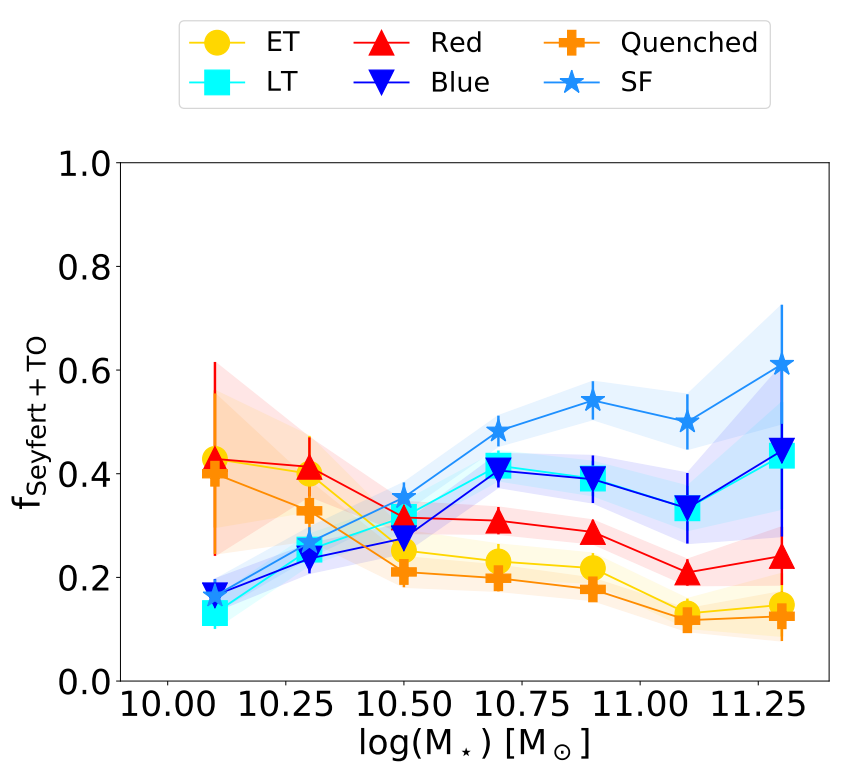

\begin{tabular}{|c|c|c|c|c|c|c|c|}
\hline ET & 6 & 18 & 31 & 36 & 44 & 18 & 5 \\
\hline LT & 16 & 53 & 76 & 118 & 84 & 40 & 10 \\
\hline Red & 3 & 31 & 71 & 95 & 109 & 53 & 14 \\
\hline Blue & 23 & 52 & 65 & 91 & 44 & 16 & 4 \\
\hline Q & 4 & 22 & 40 & 49 & 55 & 25 & 6 \\
\hline SF & 22 & 61 & 96 & 137 & 98 & 44 & 11 \\
\hline
\end{tabular}

Fig. 6. Similar to the middle panel in Fig. 2 but considering only TO and Seyfert as AGN subtypes (i.e. excluding SIG galaxies classified as LINER). The number of galaxies in each stellar mass bin is shown in the table at the bottom. Error bars are given by considering binomial distribution.

and star-forming SIG galaxies, which is higher than the fraction in early-type, red, and quenched SIG galaxies at higher masses. However we observe that this happens at slightly smaller stellar mass $\left(\log \left(M_{\star}\right)>10.5 M_{\odot}\right)$. This value was also considered to separate between low- and high-mass galaxies when exploring the effect of the LSS environment on the fraction of AGNs (see Appendix B), and it is therefore considered in the range of uncertainties. The most important difference when excluding LINER galaxies is the slow decrement in the fraction of AGNs in earlytype, red, and quenched galaxies with stellar mass, instead of being roughly constant.

We note that TOs have not been excluded from the AGN classification, since they contain an AGN component making up to $40 \%$ of their $\mathrm{H} \alpha$ luminosity (Brinchmann et al. 2004). Given that our analysis is based on spectroscopic data from the $3^{\prime}$ fibre at the central area of the galaxies, which typically contains the nuclear region at the redshift range considered in our galaxy sample, we can consider that the effect of non-AGN emission on our classification is minimal. However, we additionally explore the AGN prevalence in each AGN subtype, that is, TOs, Seyferts, and LINERs. The results of this analysis are presented in Appendix A. Even if the statistic is low, the general trend for late-type, blue, and star-forming galaxies is to increase with the stellar mass (see Fig. A.1), in agreement with the results shown in the middle panel of Fig. 2. The TO and Seyfert prevalence for early-type, red, and quenched SIG galaxies is roughly constant, also in agreement with the previous results. Only when considering LINERs do we observe an increment with stellar mass. However, the fractions of LINERs at low mass for early-type, red, and quenched galaxies are significantly larger than those for late-type, blue, and star-forming galaxies, in agreement with the previous results. Therefore, our main conclusions are valid overall.

\subsection{Dependence on the LSS environment}

Argudo-Fernández et al. (2016) found a strong dependence of the LSS on the optical nuclear activity and star formation in isolated galaxies, where the observed trends are different depending on galaxy mass. In particular, they found an increment of the fraction of low-mass SFN isolated galaxies with denser LSS. This trend is reproduced by low-mass star-forming isolated galaxies (see the upper left panel in Fig. 5), and is even steeper if we select a mass cut of $\log \left(M_{\star}\right)=10.5 M_{\odot}$ (see the upper left panel in Fig. B.1) instead of the selected $\log \left(M_{\star}\right)=10.6 M_{\odot}$. Surprisingly, we also observe this trend for low-mass red isolated galaxies (see the upper right panel in Fig. 4), even if there is a low fraction of red SFN galaxies.

To explore this trend for these two different populations of galaxies, we have checked that low-mass, red, isolated galaxies with a SFN at high $Q_{\mathrm{LSS}}$ are also star-forming galaxies. This population of red and star-forming galaxies in the local universe is known as the "Red Misfits" (Evans et al. 2018), a transition population to the red sequence, where the quenching is dominated by internal processes rather than environmentally driven processes. It would therefore be somehow expected to find this population in isolated galaxies. However Evans et al. (2018) found that the proportion of red misfits is nearly independent of environment, and we find this population for isolated galaxies at denser LSS. These preliminary differences merit further detailed study.

We do not see any trend in SFN for low-mass isolated galaxies in terms of morphology (Fig. 3). In the case of high-mass isolated galaxies, in general, there is no dependence of the LSS on the fraction of SFN, although it seems there is a slight tendency for late-type isolated galaxies to increases with the $Q_{\mathrm{LSS}}$ (see the upper left panel in Fig. 3).

As expected, the fraction of SFNs in late-type, blue, and starforming galaxies, respectively, is generally larger than in earlytype, red, and quenched galaxies, especially in low-mass galaxies (see also the left panel in Fig. 2). With the exception of lowmass star-forming galaxies, where the fraction increases with higher $Q_{\mathrm{LSS}}$, the fractions of SFN are independent of the LSS. Therefore, even when exploring the trends observed in ArgudoFernández et al. (2016) in terms of morphology, colour, and sSFR, we still do not observe an increment of the SFN in void galaxies (defined here as SIG galaxies with low values of the $Q_{\mathrm{LSS}}$ ), as reported in Liu et al. (2015). The difference between the results of the two studies may be therefore caused by the fact that the void population of galaxies is composed of galaxies with diverse local environments, commonly known as field galaxies, which may be composed of pairs and small groups of galaxies, where the spectral properties may be different and be affected by the group environment and/or interactions.

Argudo-Fernández et al. (2016) also found an increment of the fraction of AGN in high-mass SIG galaxies, and a decrement in low-mass galaxies, with denser LSS. Our results reproduce these trends in terms of colour and SSFR, in particular for highmass red and quenched isolated galaxies, and low-mass blue and star-forming isolated galaxies, respectively (see the middle panels in Figs. 4 and 5). Moreover, the increment of the fraction of AGNs with denser LSS seems to be independent of the stellar mass for both red and quenched galaxies. In addition, we also observe the fraction of AGNs in high-mass early-type 
isolated galaxies slightly increases with $Q_{\mathrm{LSS}}$ (see the right middle panel in Fig. 3). On the contrary, the fraction of AGNs in high-mass late-type, blue, and star-forming isolated galaxies does not depend on the LSS environment. We can therefore conclude that the AGN fractions for late-type, blue, and starforming isolated galaxies depend strongly on the stellar mass, that is, AGNs are "mass triggered".

Regarding passive galaxies, the results are in agreement with Argudo-Fernández et al. (2016), where the general trend of the fraction of passive isolated galaxies is to decrease with higher values of $Q_{\mathrm{LSS}}$, that is, from voids to denser regions such as clusters and filaments. This trend is also independent of galaxy mass, morphology, colour, and sSFR.

As introduced in Sect. 2.2, the classification of AGN based on BPT diagrams is not perfect and there might be some issues to take into consideration. The obtained results, in comparison with Argudo-Fernández et al. (2016), show that a separation between quenched and star-forming galaxies in terms of the SSFR provides a clean selection of SFN and AGN galaxy populations, since some star-forming galaxies could be classified as AGNs using BPT diagrams. We also note the sensitivity of the stellar mass cut $\log \left(M_{\star}\right)=10.6 M_{\odot}$ on the fraction of AGNs in terms of sSFR when separating between low-mass and highmass galaxies (see Appendix B for results using other stellar mass cuts). On the other hand, the observed trends in terms of morphology and colour are robust. The mass triggered AGNs are still dominant in late-type, blue, and star-forming SIG galaxies for different stellar mass cuts.

We also found that, in terms of colour, isolated galaxies are sensitive to the LSS, where we can also confirm that the fraction of AGNs increases with denser LSS. This is more evident in red galaxies, where this increment is also independent of the stellar mass. A similar dependence on the environment in terms of colour was reported by Deng et al. (2011) for SFR and SSFR, where the dependence is stronger for red galaxies. In the present work we report for the first time the dependence of the LSS environment on optical AGN in isolated galaxies as a function of galaxy colour, independently of the stellar mass. The results presented here suggest that the secular processes that trigger AGNs in red and quenched isolated galaxies, and partially for early-type galaxies where the trends are slightly more noisy, are especially sensitive to the LSS, that is, AGNs are "environment triggered"; their fraction is increased, and they are probably being fed by the accretion of cold gas from the LSS.

\section{Summary and conclusions}

Galaxy mass and environment both play a major role in the evolution of galaxies (Peng et al. 2010, 2012). At the same time, AGNs have been proposed as a plausible physical mechanism of the evolution of star-forming galaxies in quenched galaxies. Accordingly, we try to identify the connections between AGN activity, stellar mass, and the large-scale environment in a population of isolated galaxies, where AGNs would not be triggered by recent galaxy interactions or mergers, and thus black hole growths by secular processes (Hirschmann et al. 2013). In this work we extend a previous study carried out by ArgudoFernández et al. (2016) and we focus on isolated galaxies to investigate the effect of both mass and environment on the fraction of optical nuclear activity in isolated galaxies as a function of their morphology, colour, and sSFR.

We selected galaxies in the SDSS-based catalogue of isolated galaxies (SIG, Argudo-Fernández et al. 2015b). We used the AGN classification provided by Sabater et al. (2013) based on BPT diagnosis diagrams, where AGN are composed of TO, Seyfert, and LINER galaxies. We divided our galaxy sample in terms of morphology (provided by Huertas-Company et al. 2011), colour, and sSFR (according to the relations for SDSS galaxies provided by Lacerna et al. 2014). To quantify the effect of the LSS around SIG galaxies, we used the tidal strength exerted by all the neighbour galaxies in a volume of $5 \mathrm{Mpc}$ field radius within $500 \mathrm{~km} \mathrm{~s}^{-1}$ line-of-sight velocity difference (Argudo-Fernández et al. 2014, 2015b).

In Sect. 3.1 we explored the connection between AGNs and stellar mass when separating isolated galaxies in terms of morphology, colour, and sSFR. In Sect. 3.2 we separated galaxies in terms of stellar mass into low- and high-mass galaxies $\left(10.0 \leq \log \left(M_{\star}\right)<10.6 M_{\odot}\right.$ and $10.6 \leq \log \left(M_{\star}\right) \leq 11.4 M_{\odot}$, respectively) to explore where AGN activity is affected by the LSS.

Our findings can be summarised as follows.

We found that AGNs are strongly affected by stellar mass in "active" galaxies (namely late-type, blue, and star-forming), but are unaffected in "quiescent" galaxies (namely early-type, red, and quenched).

In agreement with Argudo-Fernández et al. (2016), the fraction of SFNs decreases with stellar mass and the fraction of AGNs increases; in the present study, however, we only observe this trend in "active" isolated galaxies.

On the contrary, when an isolated galaxy is in a quiescent state, the fractions of SFN, AGN, and passive isolated galaxies are independent of the stellar mass.

Active galactic nuclei are more prevalent in low-mass "quiescent" isolated galaxies than in late-type, blue, or star-forming isolated galaxies of the same mass. Once an isolated galaxy reaches a stellar mass of about $\log \left(M_{\star}\right) \sim 10.6 M_{\odot}$, the probability that it hosts an AGN is independent of its morphology, colour, or sSFR.

In general, the trends previously found in Argudo-Fernández et al. (2016) with respect to the LSS are weakly reproduced for "active" isolated galaxies but well reproduced by "quiescent" galaxies in terms of sSFR and colour. We do not find a clear effect of the LSS in the fraction of AGNs as a function of galaxy morphology.

In comparison to Argudo-Fernández et al. (2016), we find an increment of the fraction of SFN with denser LSS in low-mass star forming and red isolated galaxies. We find that these lowmass red galaxies with a SFN at high $Q_{\text {LSS }}$ are also star-forming galaxies, which would be considered in the population of red misfits (Evans et al. 2018).

Regarding AGNs, we find a clear increment of the fraction of AGNs with denser environment in quenched and red isolated galaxies, independently of the stellar mass. However, as opposed to what is observed in low-mass blue and star-forming SIG galaxies, we do not find a decrement of the fraction of AGNs in low-mass red and quenched galaxies.

This clear separability of the effects of environment and stellar mass on nuclear activity suggests that there are two distinct processes at work. Therein, AGN activity appears to be "mass triggered" in "active" isolated galaxies. This means that AGNs are independent of the intrinsic properties of galaxies, except for their stellar mass. On the other hand, AGNs appear to be "environment triggered" in "quiescent" isolated galaxies, where the fraction of AGNs in terms of sSFR and colour increases from void regions to denser LSS, independently of stellar mass.

Acknowledgements. The authors acknowledge the anonymous referee for his/her report, which helped to clarify and improve the quality of this work. 
This work was supported by CONICYT Astronomy Program CAS-CONICYT project No. CAS17002. This work is sponsored by the Chinese Academy of Sciences (CAS), through a grant to the CAS South America Center for Astronomy (CASSACA) in Santiago, Chile. MAF is grateful for financial support from CONICYT FONDECYT project No. 3160304. IL acknowledges partial financial support from PROYECTO FONDECYT REGULAR 1150345. SDP acknowledge financial support from the Spanish Ministerio de Economìa y Competitividad under grant AYA2013-47742-C4-1-P, AYA2016-79724-C4-4-P from the Spanish PNAYA, and from Junta de Andalucía Excellence Project PEX2011FQM-7058. This research made use of ASTROPY, a community-developed core PYTHON (http: //www . python . org) package for Astronomy (Astropy Collaboration 2013); IPYTHON (Pérez \& Granger 2007); MATPLOTLIB (Hunter 2007); NUMPY (Walt et al. 2011); scipy (Jones et al. 2001); and topCat (Taylor 2005). Funding for SDSS-III has been provided by the Alfred P. Sloan Foundation, the Participating Institutions, the National Science Foundation, and the U.S. Department of Energy Office of Science. The SDSS-III web site is http://www . sdss3. org/. SDSS-III is managed by the Astrophysical Research Consortium for the Participating Institutions of the SDSS-III Collaboration including the University of Arizona, the Brazilian Participation Group, Brookhaven National Laboratory, University of Cambridge, University of Florida, the French Participation Group the German Participation Group, the Instituto de Astrofisica de Canarias, the Michigan State/Notre Dame/JINA Participation Group, Johns Hopkins University, Lawrence Berkeley National Laboratory, Max Planck Institute for Astrophysics, New Mexico State University, New York University, Ohio State University, Pennsylvania State University, University of Portsmouth, Princeton University, the Spanish Participation Group, University of Tokyo, University of Utah, Vanderbilt University, University of Virginia, University of Washington, and Yale University.

\section{References}

Abazajian, K. N., Adelman-McCarthy, J. K., Agüeros, M. A., et al. 2009, ApJS 182,543

Ahn, C. P., Alexandroff, R., Allende Prieto, C., et al. 2014, ApJS, 211, 17 Alam, S., Albareti, F. D., Allende Prieto, C., et al. 2015, ApJS, 219, 12 Argudo-Fernández, M., Verley, S., Bergond, G., et al. 2013, A\&A, 560, A9 Argudo-Fernández, M., Verley, S., Bergond, G., et al. 2014, A\&A, 564, A94 Argudo-Fernández, M., Duarte Puertas, S., Verley, S., Sabater, J., \& Ruiz J. E. 2015a, LSSGALPY: Visualization of the large-scale environment around galaxies on the 3D space, Astrophysics Source Code Library, [record ascl:1505.012

Argudo-Fernández, M., Verley, S., Bergond, G., et al. 2015b, A\&A, 578, A110 Argudo-Fernández, M., Shen, S., Sabater, J., et al. 2016, A\&A, 592, A30

Argudo-Fernández, M., Duarte Puertas, S., Ruiz, J. E., et al. 2017, PASP, 129 , 058005

Astropy Collaboration (Robitaille, T. P., et al.) 2013, A\&A, 558, A33

Bait, O., Barway, S., \& Wadadekar, Y. 2017, MNRAS, 471, 2687

Baldwin, J. A., Phillips, M. M., \& Terlevich, R. 1981, PASP, 93, 5

Belfiore, F., Maiolino, R., Maraston, C., et al. 2016, MNRAS, 461, 3111

Best, P. N., Kauffmann, G., Heckman, T. M., et al. 2005, MNRAS, 362, 25

Blanton, M. R., \& Roweis, S. 2007, AJ, 133, 734

Bluck, A. F. L., Mendel, J. T., Ellison, S. L., et al. 2014, MNRAS, 441, 599

Bongiorno, A., Schulze, A., Merloni, A., et al. 2016, A\&A, 588, A78

Bower, R. G., Benson, A. J., Malbon, R., et al. 2006, MNRAS, 370, 645

Bremer, M. N., Phillipps, S., Kelvin, L. S., et al. 2018, MNRAS, 476, 12

Brinchmann, J., Charlot, S., White, S. D. M., et al. 2004, MNRAS, 351, 1151

Casado, J., Ascasibar, Y, Gavilán, M. et al. 2015, MNRAS, 451, 888

Catalán-Torrecilla, C., Gil de Paz, A., Castillo-Morales, A., et al. 2017, ApJ, 848, 87

Cheung, E., Bundy, K., Cappellari, M., et al. 2016, Nature, 533, 504

Chiaberge, M., Gilli, R., Lotz, J. M., \& Norman, C. 2015, ApJ, 806, 147

Coenda, V., Martínez, H. J., \& Muriel, H. 2018, MNRAS, 473, 5617

Cora, S. A., Hough, T., Vega-Martínez, C. A., \& Orsi, 2018, MNRAS, submitted, [1801.03884]

Croton, D. J., Springel, V., White, S. D. M., et al. 2006, MNRAS, 365, 11

Deng, X.-F., Chen, Y.-Q., \& Jiang, P. 2011, MNRAS, 417, 453

Dressler, A. 1980, ApJ, 236, 351

Duarte Puertas, S., Vilchez, J. M., Iglesias-Páramo, J., et al. 2017, A\&A, 599, A71

Ellison, S. L., Patton, D. R., Mendel, J. T., \& Scudder, J. M. 2011, MNRAS, 418, 2043

Evans, F. A., Parker, L. C., \& Roberts, I. D. 2018, MNRAS, 476, 5284

Fraser-McKelvie, A., Pimbblet, K. A., Penny, S. J., \& Brown, M. J. I. 2016, MNRAS, 459, 754

Gaspari, M., Brighenti, F., \& Temi, P. 2012, MNRAS, 424, 190

Harrison, C. M. 2017, Nat. Astron., 1, 0165

Heckman, T. M. 1980, A\&A, 87, 152

Heckman, T. M., \& Best, P. N. 2014, ARA\&A, 52, 589
Hernández-Toledo, H. M., Vázquez-Mata, J. A., Martínez-Vázquez, L. A., Choi, Y.-Y., \& Park, C. 2010, AJ, 139, 2525

Hernández-Ibarra, F. J., Krongold, Y., Dultzin, D., et al. 2016, MNRAS, 459, 291

Hernquist, L. 1993, ApJ, 409, 548

Hirschmann, M., De Lucia, G., Iovino, A., \& Cucciati, O. 2013, MNRAS, 433, 1479

Hirschmann, M., De Lucia, G., Wilman, D., et al. 2014, MNRAS, 444, 2938

Hong, J., Im, M., Kim, M., \& Ho, L. C. 2015, ApJ, 804, 34

Huertas-Company, M., Aguerri, J. A. L., Bernardi, M., Mei, S., \& Sánchez Almeida, J. 2011, A\&A, 525, A157

Hunter, J. D. 2007, Comput. Sci. Eng., 9, 90

Hwang, H. S., Park, C., Elbaz, D., \& Choi, Y.-Y. 2012, A\&A, 538, A15

Jones, E., Oliphant, T., Peterson, P., et al. 2001, SciPy: Open source scientific tools for Python

Kauffmann, G. 1996, MNRAS, 281, 487

Kauffmann, G., Heckman, T. M., Tremonti, C., et al. 2003a, MNRAS, 346, 1055

Kauffmann, G., Heckman, T. M., White, S. D. M., et al. 2003b, MNRAS, 341, 33

Kauffmann, G., White, S. D. M., Heckman, T. M., et al. 2004, MNRAS, 353, 713

Kaviraj, S., Shabala, S. S., Deller, A. T., \& Middelberg, E. 2015, MNRAS, 452, 774

Lacerna, I., Rodríguez-Puebla, A., Avila-Reese, V., \& Hernández-Toledo, H. M. 2014, ApJ, 788, 29

Lacerna, I., Hernández-Toledo, H. M., Avila-Reese, V., Abonza-Sane, J., \& del Olmo, A. 2016, A\&A, 588, A79

Lacerna, I., Argudo-Fernández, M., \& Duarte Puertas, S. 2018, A\&A, in press, DOI: $10.1051 / 0004-6361 / 201833579$

Liu, C.-X., Pan, D. C., Hao, L., et al. 2015, ApJ, 810, 165

Manzer, L. H., \& De Robertis, M. M. 2014, ApJ, 788, 140

McAlpine, K., Prandoni, I., Jarvis, M., et al. 2015, Advancing Astrophysics with the Square Kilometre Array (AASKA14), 83,

Melnyk, O., Karachentseva, V., \& Karachentsev, I. 2015, MNRAS, 451, 1482

Moles, M., Marquez, I., \& Perez, E. 1995, ApJ, 438, 604

Noeske, K. G., Weiner, B. J., Faber, S. M., et al. 2007, ApJ, 660, L43

Nogueira-Cavalcante, J. P., Gonçalves, T. S., Menéndez-Delmestre, K., \& Sheth, K. 2018, MNRAS, 473, 1346

Peng, Y., Maiolino, R., \& Cochrane, R. 2015, Nature, 521, 192

Peng, Y.-J., Lilly, S. J., Kovač, K., et al. 2010, ApJ, 721, 193

Peng, Y.-J., Lilly, S. J., Renzini, A., \& Carollo, M. 2012, ApJ, 757, 4

Penny, S. J., Masters, K. L., Weijmans, A.-M., et al. 2016, MNRAS, 462, 3955

Penny, S. J., Masters, K. L., Smethurst, R., et al. 2018, MNRAS, 476, 979

Pérez, F., \& Granger, B. E. 2007, Comput. Sci. Eng., 9, 21

Ramos Almeida, C., Bessiere, P. S., Tadhunter, C. N., et al. 2012, MNRAS, 419, 687

Renzini, A., \& Peng, Y.-J. 2015, ApJ, 801, L29

Richards, S. N., Bryant, J. J., Croom, S. M., et al. 2016, MNRAS, 455, 2826

Rodighiero, G., Daddi, E., Baronchelli, I., et al. 2011, ApJ, 739, L40

Rodríguez del Pino, B., Aragón-Salamanca, A., Chies-Santos, A. L., et al. 2017, MNRAS, 467, 4200

Rosario, D. J., Mendel, J. T., Ellison, S. L., Lutz, D., \& Trump, J. R. 2016, MNRAS, 457, 2703

Rowlands, K., Wild, V., Bourne, N., et al. 2018, MNRAS, 473, 1168

Sabater, J., Leon, S., Verdes-Montenegro, L., et al. 2008, A\&A, 486, 73

Sabater, J., Verdes-Montenegro, L., Leon, S., Best, P., \& Sulentic, J. 2012, A\&A, 545, A15

Sabater, J., Best, P. N., \& Argudo-Fernández, M. 2013, MNRAS, 430, 638

Sabater, J., Best, P. N., \& Heckman, T. M. 2015, MNRAS, 447, 110

Salim, S., Rich, R. M., Charlot, S., et al. 2007, ApJS, 173, 267

Satyapal, S., Ellison, S. L., McAlpine, W., et al. 2014, MNRAS, 441, 1297

Schawinski, K., Dowlin, N., Thomas, D., Urry, C. M., \& Edmondson, E. 2010 ApJ, 714, L108

Schawinski, K., Urry, C. M., Simmons, B. D., et al. 2014, MNRAS, 440, 889

Silk, J., \& Rees, M. J. 1998, A\&A, 331, L1

Singh, R., van de Ven, G., Jahnke, K., et al. 2013, A\&A, 558, A43

Smethurst, R. J., Lintott, C. J., Simmons, B. D., et al. 2016, MNRAS, 463, 2986

Smethurst, R. J., Masters, K. L., Lintott, C. J., et al. 2018, MNRAS, 473, 2679

Strauss, M. A., Weinberg, D. H., Lupton, R. H., et al. 2002, AJ, 124, 1810

Taylor, M. B. 2005, in Astronomical Data Analysis Software and Systems XIV, eds. P. Shopbell, M. Britton \& R. Ebert, ASP Conf. Ser., 347, 29

Terrazas, B. A., Bell, E. F., Woo, J., \& Henriques, B. M. B. 2017, ApJ, 844, 170

Tremonti, C. A., Heckman, T. M., Kauffmann, G., et al. 2004, ApJ, 613, 898

Tutukov, A. V., Dryomov, V. V., \& Dryomova, G. N. 2007, Astron. Rep., 51, 435

Verley, S., Leon, S., Verdes-Montenegro, L., et al. 2007, A\&A, 472, 121

Vulcani, B., Poggianti, B. M., Fritz, J., et al. 2015, ApJ, 798, 52

Walt, S. v. d., Colbert, S. C., \& Varoquaux, G. 2011, Comput. Sci. Eng., 13, 22 


\section{Appendix A: AGN subtypes prevalence}

The AGN classification used in this study, adopted from Sabater et al. (2013), is composed of galaxies classified as TO, Seyfert, and LINER. In Sect. 3.1 we studied the optical AGN prevalence of isolated galaxies with respect to stellar mass, as a function of morphology, colour, and sSFR. To explore the effect of the AGN classification in our conclusions, in this section we compare the relative fraction of each AGN subtype in the same stellar mass bins.

The three panels of Fig. A.1 show the fractions of SIG galaxies classified as TO, Seyfert, and LINER. Therefore, at fixed stellar mass, the sum of each number in the lower tables is equal to the number of AGNs in the lower table of the middle panel in Fig. 2 for the same galaxy group. For instance, the sum of the number of isolated early-type galaxies classified as TO
(5 galaxies), Seyfert (1 galaxy), and LINER (1 galaxy) in the first mass bin is equal to 7 , as shown in the table of the middle panel in Fig. 2.

The trend observed in the AGN fractions of massive galaxies, $\log \left(\mathrm{M}_{\star}\right)>10.6 M_{\odot}$, in the middle panel of Fig. 2 is qualitatively reproduced by "active" (late-type, blue, and star-forming) SIG galaxies with equal or larger fractions of TOs and Seyferts than "passive" (early-type, red, and quenched) SIG galaxies. In the case of LINERs, the fraction is slightly lower in active galaxies than in passive galaxies. For lower stellar masses, the middle panel of Fig. 2 shows an overall trend where the fraction of each AGN subtype in passive galaxies is equal to or larger than those in active galaxies. This trend, although noisier, is still observable when we separate the AGN classification into the three subtypes.

The possible consequences of these results on our main conclusions are discussed in Sect. 4.1.

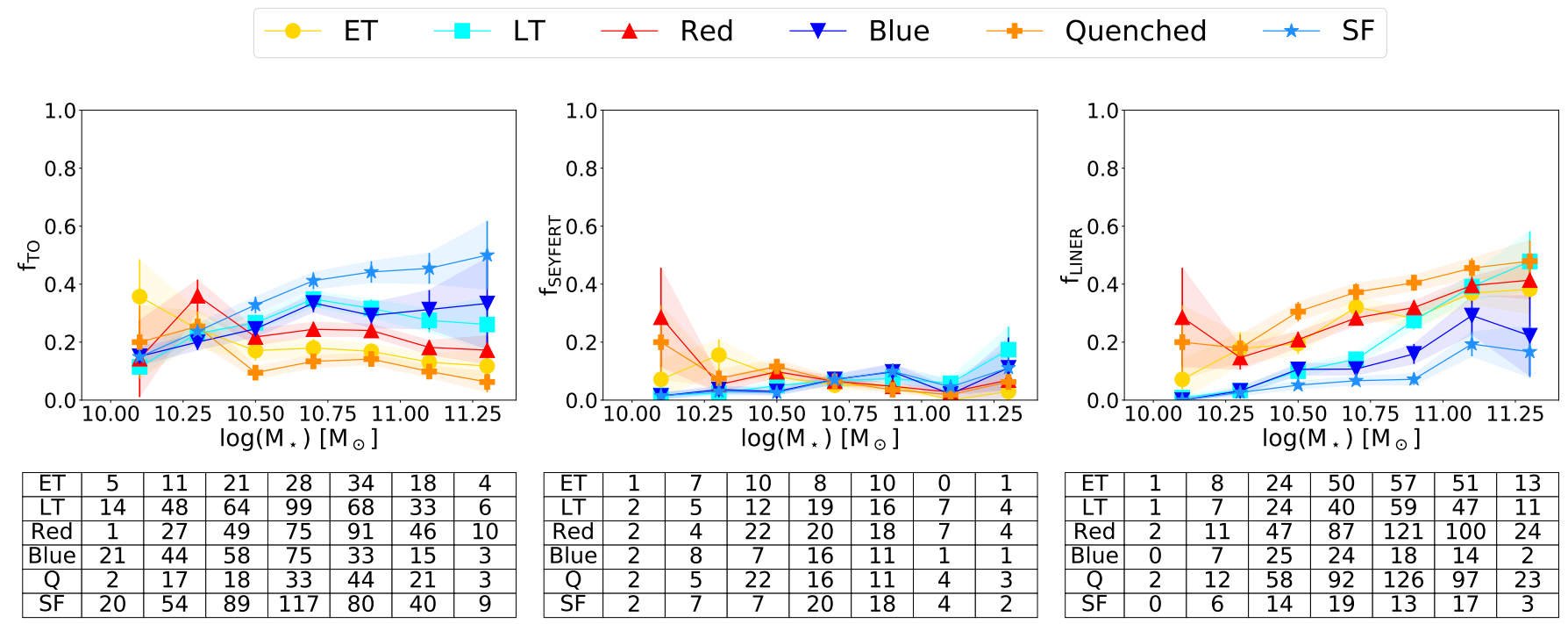

Fig. A.1. Fraction of TO (left panel), Seyfert (middle panel), and LINER galaxies (right panel) with respect to stellar mass. Following Fig. 2, the fraction in early-type $\left(N_{T}=362\right)$, red $\left(N_{T}=768\right)$, and quenched $\left(N_{T}=611\right)$ SIG galaxies classified as AGN are depicted by yellow circles, red triangles, and orange pluses, respectively. Cyan squares correspond to the fraction of AGN in late-type SIG galaxies $\left(N_{T}=586\right)$, blue inverted triangles for blue SIG galaxies $\left(N_{T}=385\right)$, and light blue stars for star-forming SIG galaxies $\left(N_{T}=541\right)$. The fractions of TO $(l e f t$ panel $)$, Seyfert (middle panel), and LINER (right panel) galaxies are shown in tables for each sample at the bottom of each panel. Error bars are given by considering binomial distribution. 


\section{Appendix B: Effect of the selected stellar mass cut}

According to the distributions presented in Fig. 1, we selected a stellar mass cut $\log \left(M_{\star}\right)=10.6 M_{\odot}$ as a good compromise between the number of galaxies at each side of the distributions and an equally valid value when separating between late- and early-type galaxies, blue and red galaxies, and star-forming and quenched galaxies, respectively.

However, when exploring the AGN prevalence as a function of stellar mass, we observe that there is a transition stellar mass range around the selected mass cut $\log \left(M_{\star}\right)=10.6 M_{\odot}$, where the fractions of AGN in "active" isolated galaxies equal the fractions of AGN in "quiescent" isolated galaxies (see the middle panel in Fig. 2).

Here we explore whether there is any effect of the stellar mass cut value on the observed trends of the fractions of SFN, AGN, and passive galaxies with the LSS presented in Sect. 4.2. We select $\log \left(M_{\star}\right)=10.5 M_{\odot}$ and $\log \left(M_{\star}\right)=10.7 M_{\odot}$ as low and high limit values, respectively, of the critical stellar mass region.

Figures B. 1 and B. 2 are similar to Fig. 5 but using a stellar mass cut $\log \left(M_{\star}\right)=10.5 M_{\odot}$ and $\log \left(M_{\star}\right)=10.7 M_{\odot}$, respectively. We observe that the trends of the fractions of AGN for starforming and quenched galaxies are somewhat sensitive to the stellar mass cut. In particular, using a mass cut $\log \left(M_{\star}\right)=10.5 M_{\odot}$ the observed trends in low-mass star-forming galaxies are steeper. We also find a separation of the fraction of AGN for the highest bin of $Q_{\text {LSS }}$ in quenched galaxies, where the fraction of AGN in low-mass galaxies is lower than the fraction of AGN in high-mass galaxies. Since this observed separation disappear when selecting $\log \left(M_{\star}\right)=10.6 M_{\odot}$ and $\log \left(M_{\star}\right)=10.7 M_{\odot}$, it may be an artefact of the low number of low-mass quenched galaxies in that bin. Similarly on the fraction of passive quenched galaxies. We do not observe any difference in the trends in terms of morphology and colour and therefore do not show the corresponding figures.
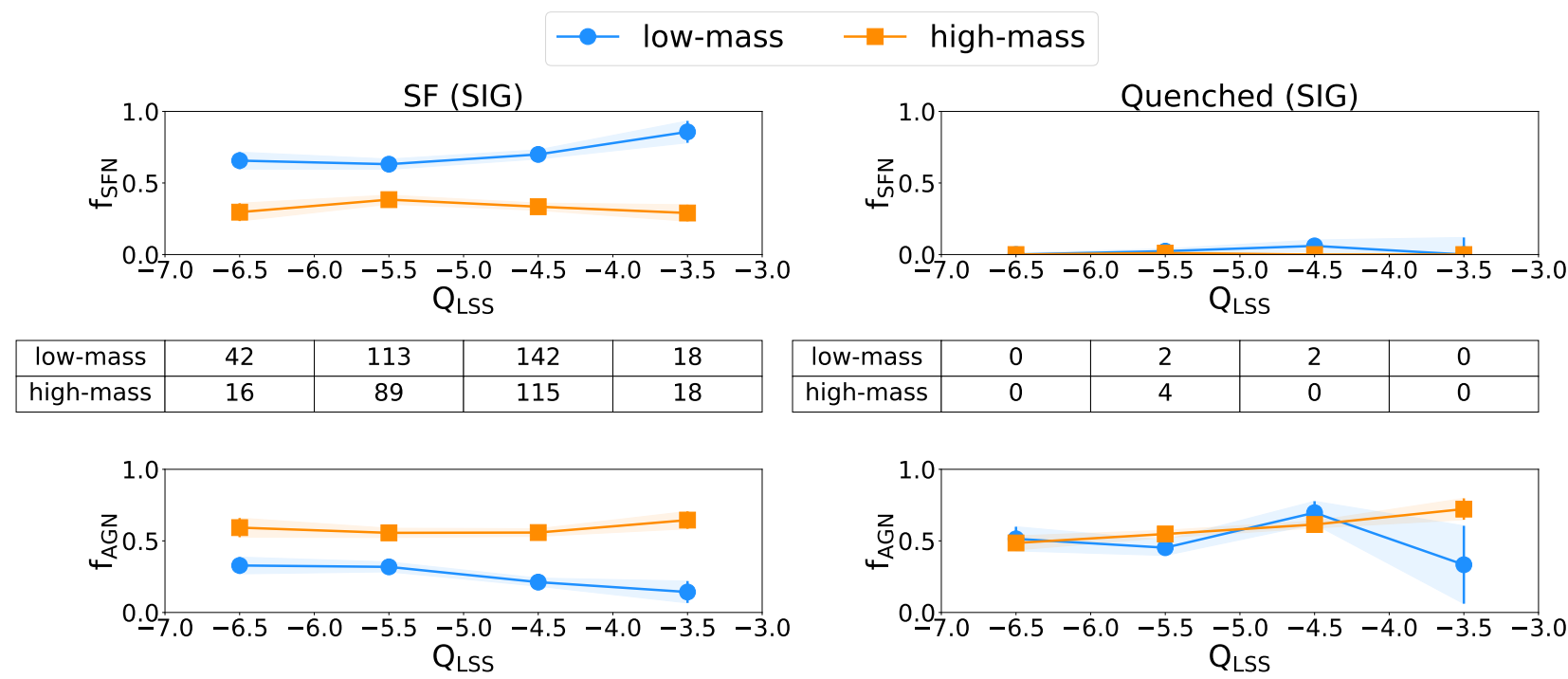

\begin{tabular}{|c|l|l|l|l|}
\hline low-mass & 0 & 2 & 2 & 0 \\
\hline high-mass & 0 & 4 & 0 & 0 \\
\hline
\end{tabular}

\begin{tabular}{|c|c|c|c|c|}
\hline low-mass & 21 & 57 & 43 & 3 \\
\hline high-mass & 32 & 129 & 192 & 40 \\
\hline
\end{tabular}
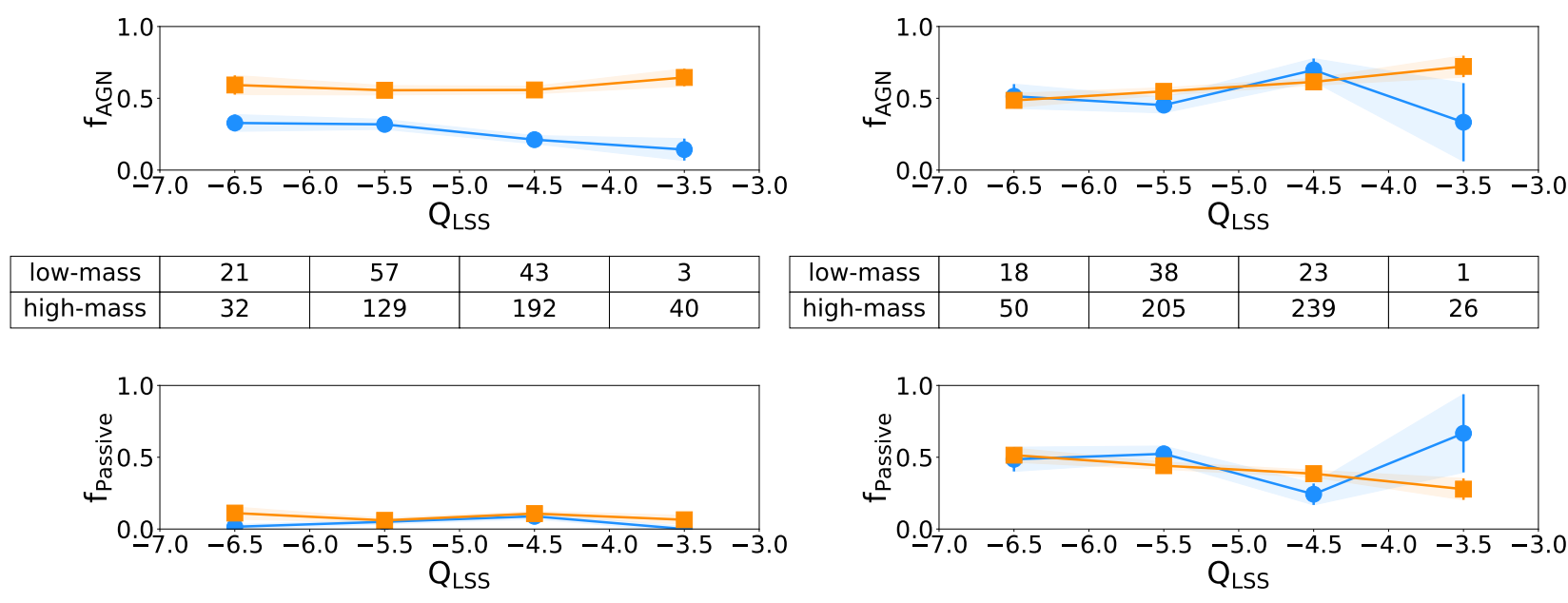

\begin{tabular}{|c|c|c|c|c|}
\hline low-mass & 18 & 38 & 23 & 1 \\
\hline high-mass & 50 & 205 & 239 & 26 \\
\hline
\end{tabular}

\begin{tabular}{|c|c|c|c|c|}
\hline low-mass & 1 & 9 & 18 & 0 \\
\hline high-mass & 6 & 14 & 37 & 4 \\
\hline
\end{tabular}

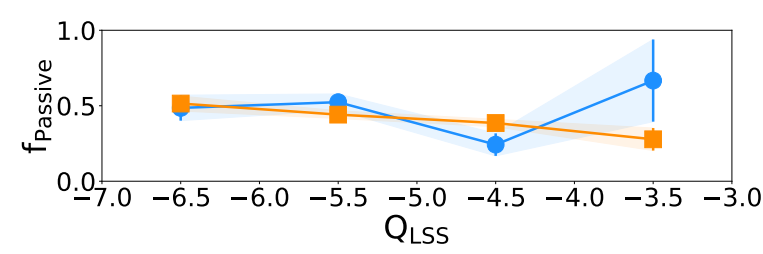

\begin{tabular}{|c|c|c|c|c|}
\hline low-mass & 17 & 44 & 8 & 2 \\
\hline high-mass & 53 & 165 & 150 & 10 \\
\hline
\end{tabular}

Fig. B.1. Fraction of optical nuclear activity with respect to the $Q_{\text {LSS }}$ environmental parameter according to their sSFR status. Low-mass galaxies $\left(10.0 \leq \log \left(M_{\star}\right)<10.5 M_{\odot}\right)$ are represented by cyan circles, and high-mass galaxies $\left(10.5 \leq \log \left(M_{\star}\right) \leq 11.4 M_{\odot}\right)$ are represented by orange squares. The fraction of SFN, optical AGN, and passive star forming SIG galaxies ( $N_{T}=577,541$, and 91, respectively) is represented from top to bottom in the left panels, and for quenched SIG galaxies $\left(N_{T}=8,611\right.$, and 468 , respectively) in the right panels. The number of galaxies in each $Q_{\mathrm{LSS}}$ bin is shown in tables for each sample at the bottom of each panel. Error bars are given considering binomial distribution. 

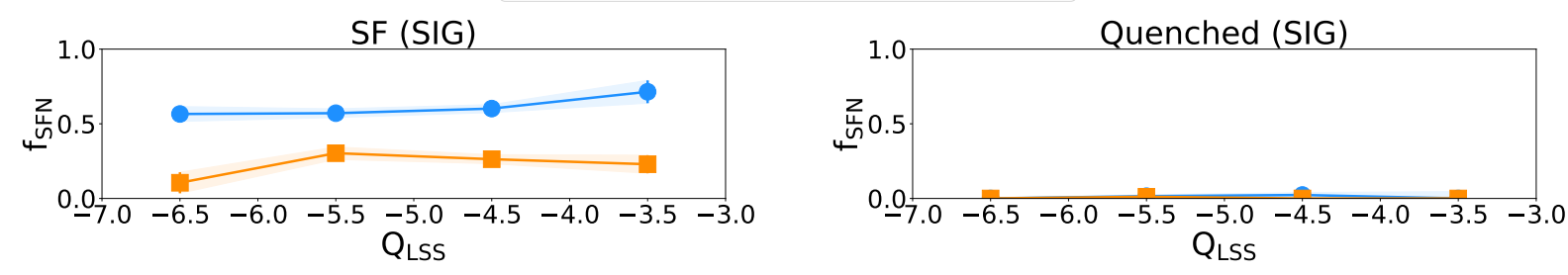

\begin{tabular}{|c|c|c|c|c|}
\hline low-mass & 56 & 165 & 201 & 25 \\
\hline high-mass & 2 & 37 & 56 & 11 \\
\hline
\end{tabular}

\begin{tabular}{|c|l|l|l|l|}
\hline low-mass & 0 & 3 & 2 & 0 \\
\hline high-mass & 0 & 3 & 0 & 0 \\
\hline
\end{tabular}
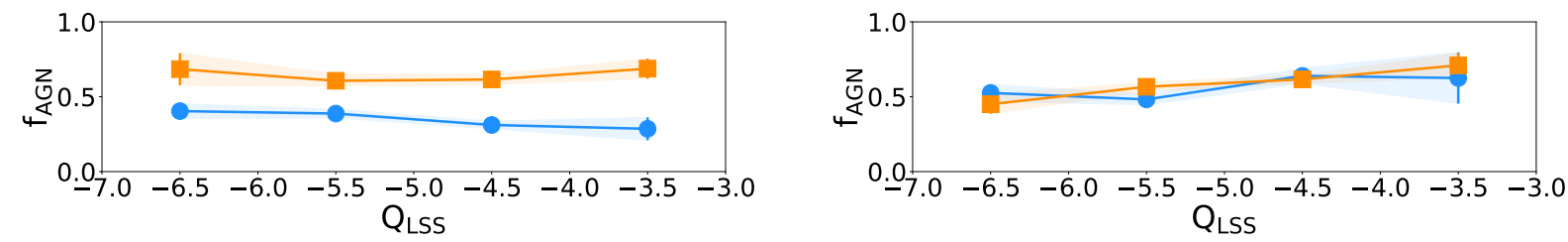

\begin{tabular}{|c|c|c|c|c|}
\hline low-mass & 40 & 112 & 104 & 10 \\
\hline high-mass & 13 & 74 & 131 & 33 \\
\hline
\end{tabular}

\begin{tabular}{|c|c|c|c|c|}
\hline low-mass & 41 & 94 & 55 & 5 \\
\hline high-mass & 27 & 149 & 207 & 22 \\
\hline
\end{tabular}
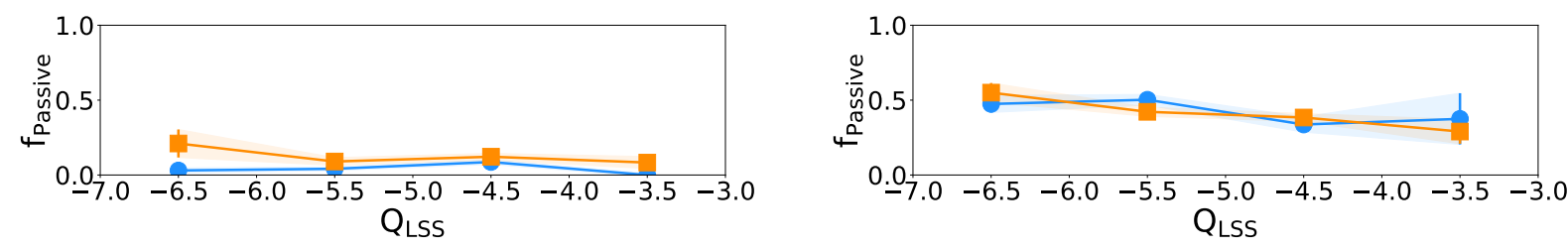

\begin{tabular}{|c|l|l|l|l|}
\hline low-mass & 3 & 12 & 29 & 0 \\
\hline high-mass & 4 & 11 & 26 & 4 \\
\hline
\end{tabular}

\begin{tabular}{|c|c|c|c|c|}
\hline low-mass & 37 & 98 & 29 & 3 \\
\hline high-mass & 33 & 111 & 129 & 9 \\
\hline
\end{tabular}

Fig. B.2. Fraction of optical nuclear activity with respect to the $Q_{\mathrm{LSS}}$ environmental parameter according to their sSFR status. Low-mass galaxies $\left(10.0 \leq \log \left(M_{\star}\right)<10.7 M_{\odot}\right)$ are represented by cyan circles, and high-mass galaxies $\left(10.7 \leq \log \left(\mathrm{M}_{\star}\right) \leq 11.4 M_{\odot}\right)$ are represented by orange squares. The fraction of SFN, optical AGN, and passive star forming SIG galaxies ( $N_{T}=577,541$, and 91, respectively) is represented from top to bottom in the left panels, and for quenched SIG galaxies $\left(N_{T}=8,611\right.$, and 468 , respectively) in the right panels. The number of galaxies in each $Q_{\mathrm{LSS}}$ bin is shown in tables for each sample at the bottom of each panel. Error bars are given considering binomial distribution. 